\title{
Immunopathophysiologic basis of multiple sclerosis and implications for therapy-a narrative review
}

\begin{abstract}
Our current understanding of multiple sclerosis (MS) recognizes several immunopathological mechanisms leading to progressive axonal loss, brain atrophy, and neurological and cognitive disability. Autoreactive T cells eluding central tolerance in the thymus enter lymph nodes. There, antigen presenting cells expressing an antigen closely resembling a self-protein or autoantigen, activate $\mathrm{CD} 4+$ cells, which then differentiate into proinflammatory $\mathrm{T}$ helper cells (Th1 and Th17). These cells release TNF-alpha, causing myelin disruption and oligodendrocyte apoptosis, and INF $\gamma$ which recruits CD8+ cells, B cells, and monocytes. These cells leave the lymph nodes and enter the CNS. CD8+ cells attack oligodendrocytes synthesizing myelin and exacerbate neuronal damage. Monocytes (macrophages) phagocytose the myelin sheath and release proinflammatory cytokines that enhance the local inflammatory response. MS progression is characterized by diffuse $\mathrm{T}$ and $\mathrm{B}$ cell infiltrates, astrogliosis and microgliosis. New evidence suggests that memory B cells may represent a principal component of MS etiology and T and B cell autoimmunity may be a secondary driver of MS. Understanding the immunopathological underpinnings of MS has revolutionized the treatment of this neurological autoimmune disease with an array of disease-modifying drugs (DMDs) with mechanisms of action directed at various key points. Ideally, DMDs should be initiated early in the disease course with the goal to reduce disease activity, preserve brain tissue, and limit deterioration in cognitive and physical function. Currently approved DMDs can be broadly categorized into platform therapies (INFs and glatiramer acetate), S1P receptor modulators, fumarates, monoclonal antibodies, and other therapies. DMDs can also be categorized by overall treatment approach: chronic maintenance/escalation therapy and immune reconstitution therapy (IRT). It remains unclear, however, whether IRT agents reset the adaptive immune system or simply result in cell types with different repopulation dynamics. Other therapeutic approaches in clinical development include Bruton's tyrosine kinase inhibitors, remyelination, neuroprotection, and modulation of microbiome triggers.
\end{abstract}

Keywords: immunology, multiple sclerosis, pathophysiology, disease modifying drugs, immunology, lesions, disability, magnetic resonance imaging

\author{
Volume 9 Issue 6 - 2021
}

\author{
Jan S Redfern \\ Redfern Strategic Medical Communications, Inc., USA
}

Correspondence: Jan S Redfern, PhD, Redfern Strategic Medical Communications, Inc., 700 Jay Bird Road Springtown, Texas 76082, United States of America, Email Jan@redfernstrategic.com

Received: December 10,2021 | Published: December 21, 2021
Abbreviations: AAN, American Academy of Neurology; $\mathrm{APC}$, antigen presenting cell; BBB, blood-brain barrier; $\mathrm{CD}$, cluster of differentiation; CNS, central nervous system; CIS, clinically isolated syndrome; DMD, disease modifying drug; DMF, dimethyl fumarate; DNA, deoxyribonucleic acid; EAN, European Academy of Neurology; EDSS, expanded disability status scale; ECTRIMS, European Committee for Treatment and Research in Multiple Sclerosis; FGF, fibroblast growth factor; FOXP3, forkhead box P3; $\mathrm{Gd}+$, gadolinium; HSCT, hematopoietic stem cell transplantation; IM, intramuscular; INF, interferon; IL-2, interleukin-2; IL-10, interleukin-10; IRT, immune reconstitution therapy; IV, intravenous; MHC, major histocompatibility complex; MMF, monomethyl fumarate; MRI, magnetic resonance imaging; MS, multiple sclerosis; NK, natural killer; NEDA, no evidence of disease activity; NFkB, nuclear factor kappa-light-chain-enhancer of activated B cells; NICE, National Institute for Health and Care Excellence; Nrf2, nuclear factor-erythroid factor 2-related factor 2; PO, per os (by mouth); RRMS, relapsing-remitting multiple sclerosis; SC, subcutaneous; SPMS, secondary progressive multiple sclerosis; PDGF, plateletderived growth factor; PPMS, primary progressive MS; RIS, radiologically isolated syndrome; S1P-R1, sphingosine-1-phosphate receptor 1; S1P-R5, sphingosine-1-phosphate receptor 5; TCR, T cell receptor; Th1, T helper cell, type 1; Th17, T helper cell type 17; $\mathrm{Th} 2$, T helper cell type 2; TNF $\alpha$, tumour necrosis factor alpha; Treg, regulatory T cell; Breg, regulatory B cell; VCAM-1, vascular cell adhesion molecule 1; VLA-4; very late antigen-4

\section{Introduction}

Jean-Martin Charcot, a French neurologist at the Hôpital de Salpétrière, Paris, France, is credited with the first clinical description of multiple sclerosis (MS) in 1868, a disease he termed "la sclérose en plaques." Approximately 2.8 million people globally suffer from MS, which is 3 times more prevalent in women than men, most often afflicts patients between 20 and 50 years of age, and represents the most common central nervous system (CNS) disease in young adults. ${ }^{2-4}$

Although its exact etiology is not completely understood, MS is generally recognized to be an aberrant autoimmune-mediated, progressive inflammatory disease of the CNS, the key characteristic of which is degeneration of the myelin sheath surrounding axons and loss of oligodendrocytes. ${ }^{3,5} \mathrm{MS}$ is thought to be triggered by environmental factors or gut microbiome in genetically susceptible individuals. ${ }^{5}$ Demyelination (a pathologic hallmark of MS) leads to a complex cascade of inflammatory processes resulting in axonal loss and formation of focal lesions (plaque or scar tissue) present in the white matter of brain and spinal cord. ${ }^{6}$ These plaques can be visualized by magnetic resonance imaging (MRI) and correspond 
to destruction of specific brain areas and lead to intermittent or permanent neurological signs and symptoms. ${ }^{7}$ Lesions can also occur in grey matter and correlate with cognitive and clinical disability. ${ }^{7}$

MS is a heterogeneous disease with a natural history typified by clinical relapses and disease progression. ${ }^{7}$ MS can impact all aspects of daily life, although specific signs and symptoms vary from patient to patient, depending on what part of the CNS is impacted, as well as vary over time in the same patient. ${ }^{8}$ Typical symptoms include vision problems such as optic neuritis (initial presentation for many MS patients), fatigue (occurs in $80 \%$ of patients), dysesthesia, ataxia, sensory disturbance, gait disturbance, Lhermitte's sign, numbness or tingling, and muscle weakness in the extremities. ${ }^{3}$

Currently, there is no cure for MS, but it is treatable. ${ }^{9}$ The past three decades have witnessed increased understanding of the pathogenesis of multiple sclerosis, especially the central role of the immune system, both adaptive and innate. This immunopathological underpinning has revolutionized the treatment of this neurological autoimmune disease. This review serves as an introduction to 1) MS heterogeneity, clinical course, and pathophysiological mechanism of MS, 2) the central role of the adaptive and innate immune system and their interaction and compartmentalization within the CNS that drives MS disease activity, and 3) the available disease modifying drugs (DMDs) targeting effector T lymphocytes cells, regulatory cells, B lymphocytes, and trafficking of immune cells into the CNS.

\section{MS heterogeneity, clinical course, and pathophysiological mechanisms}

Overall, the general pattern of MS is one of relapses (caused by lesions in the CNS which amass over time) and subsequent disease progression. This clinical course of MS corresponds to the occurrence of two overlapping and interconnected components: inflammation and neurodegeneration (Figure 1)..$^{3,5,7,-13}$ Inflammation dominates in the early stages of the disease but neurodegenerative progression inevitably ensues in many cases. ${ }^{14}$ The peripheral immune system targeting the CNS is central to driving the disease process early on but immune reactions within the CNS dominate the progressive (neurodegeneration) phase. ${ }^{15}$

Five subtypes of MS are generally recognized: ${ }^{3} 1$ ) a preclinical type (radiologically isolated syndrome or RIS) before symptoms are evident; 2) a clinically isolated syndrome (CIS) type, resulting from inflammation and demyelination in the CNS, represents the first episode of neurological symptoms lasting at least 24 hours; 3) a relapsing-remitting (RRMS) type characterized by discrete episodes of neurological dysfunction followed by complete or partial remission; 4) a secondary progressive (SPMS) type, evolving from the relapsing-remitting type, and characterized by a progressive worsening of disability over time, and finally 5) primary progressive type (PPMS), whereby progressive worsening occurs from onset of MS without superimposed relapses.

The RRMS phase is associated with relapses (sudden attack of signs/symptoms), nominal disability, and gadolinium $(\mathrm{Gd}+)$ enhanced lesions and is primarily propelled by the adaptive immune system. ${ }^{11}$ The later, progressive course of the disease is characterized by neurodegeneration and progressive disability (with or without superimposed relapses) and is predominately driven by innate immune system. ${ }^{3,16}$ MRI Gd+ T1 lesions, which are associated with active inflammation due to blood brain barrier (BBB) breakdown and movement of immune cells into the CNS, are more frequent early on in the disease course and not always manifested as clinical relapses. ${ }^{3}$ However, MRI T2- weighted lesions, which reflect the overall disease burden or lesion load (i.e., total number of lesions, old and new), gradually increases over time and is associated with brain atrophy.
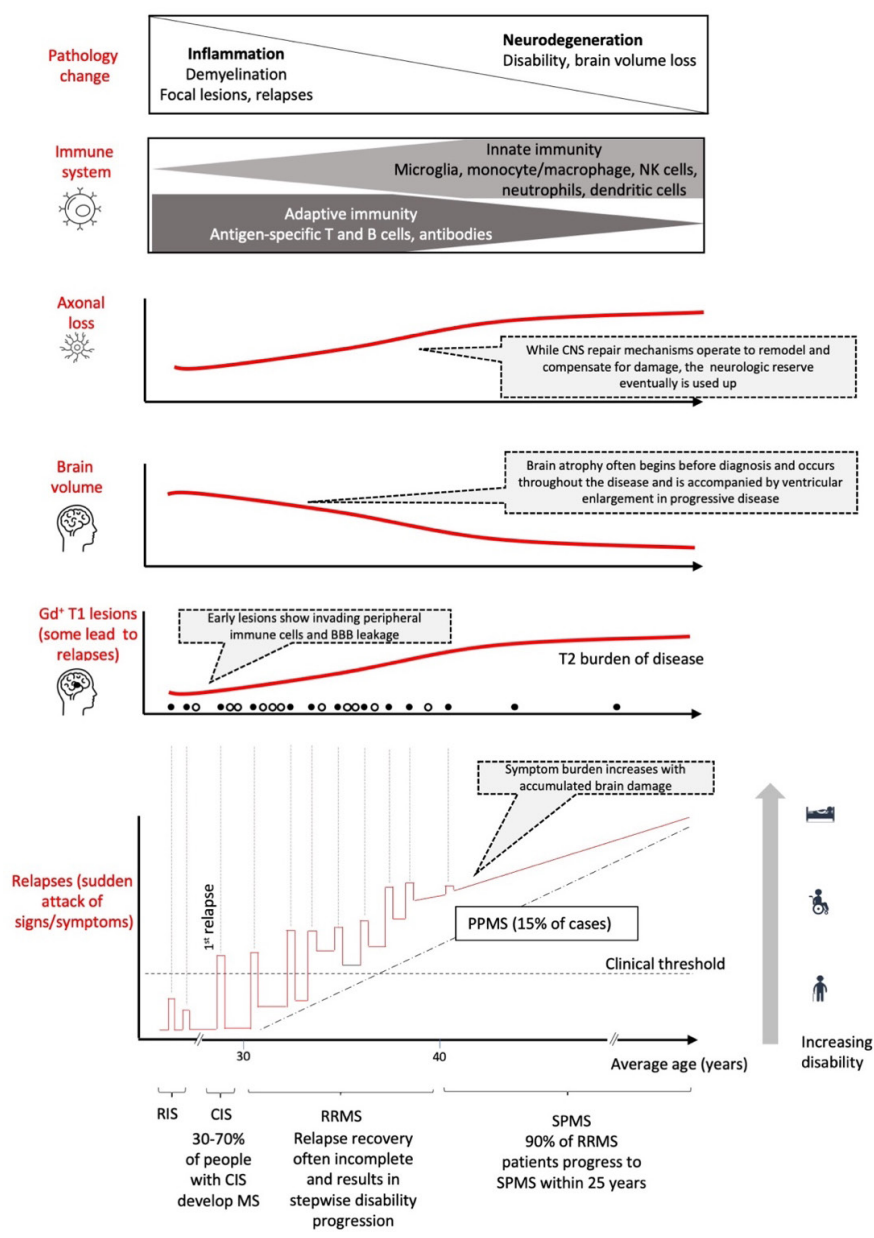

Figure I Summary of MS heterogeneity, clinical course, and pathophysiological

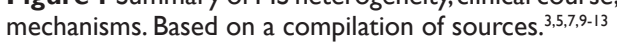

$\mathrm{BBB}$, blood brain barrier; CIS, clinically isolated syndrome; NK, natural killer; RRMS, relapsing-remitting multiple sclerosis; SPMS, secondary progressive multiple sclerosis; PPMS, primary progressive MS.

\section{Immunological basis for MS}

The immune system ordinarily exhibits self-tolerance and does not attack healthy tissues. This tolerance to self-antigens develops from a number of mechanisms including, for example, deletion of autoreactive lymphocytes (either during cell development in the thymus or bone marrow or at the mature stage in peripheral lymphoid tissues) and suppression by regulatory $\mathrm{T}$ cells (Tregs) and regulatory $\mathrm{B}$ cells (Bregs). ${ }^{17}$ Indeed, Tregs are critical in thwarting development of autoimmune diseases. ${ }^{18}$ The appearance of autoreactive T cells may be due to a defect in regulatory cells such as forkhead box P3 (FOXP3)-expressing CD4+ regulatory T cells (Treg) and IL-10producing Treg type 1 cells. ${ }^{7}$ This defect results in some autoreactive $\mathrm{T}$ cells being released peripherally where they can be activated by antigen presenting cells (in association with genetic, environmental factors or infectious agents) and contribute to development of MS (Table 1). ${ }^{19-21}$ 
Table I Proposed genetic risk factors for MS and the association with environmental factors. Based on Celarain and Tomas-Roig, 202I,Waubant E et al, 20I9, and Jakimovski, D et al 201919-21

\begin{tabular}{|c|c|c|}
\hline \multicolumn{2}{|c|}{ Environmental factors } & Genetic factors \\
\hline . & Smoking & Sex \\
\hline . & Vitamin $D$ deficiency and low sun exposure & Disease modifier genes \\
\hline . & Exposure to organic solvents/pollutants & Disease susceptibility genes (HLA DRB I5*0I allele) \\
\hline . & Diet (greater intake of butter, milk, animal fats and animal meat) & Single nucleotide polymorphism \\
\hline . & High salt intake & \\
\hline . & Epstein Barr virus exposure & \\
\hline · & Dysbiosis in the gut microbiota & \\
\hline . & Lack of physical exercise & \\
\hline . & Stress & \\
\hline
\end{tabular}

The exact immunopathological mechanisms of MS are not fully elucidated but can be simplified into a several key steps (Figure 2). ${ }^{19}$ Autoreactive T cells that have eluded central tolerance (in part, due to Treg escape) in the thymus enter the lymph nodes. Antigen presenting cells (APCs), including B cells, expressing, for example, an antigen closely resembling part of a self-protein (i.e., molecular mimicry) or putative autoantigens myelin basic protein, proteolipid protein and myelin oligodendrocyte glycoprotein via MHC class II molecules, activate naïve $\mathrm{CD} 4+\mathrm{T}$ cells. Activation of $\mathrm{CD} 4+$ requires not only antigen interaction with $\mathrm{T}$ cell receptor (TCR) on naive $\mathrm{CD} 4+\mathrm{T}$ helper cells but also co-stimulation via CD40L:CD40 and B7:CD28 interaction. $^{22}$

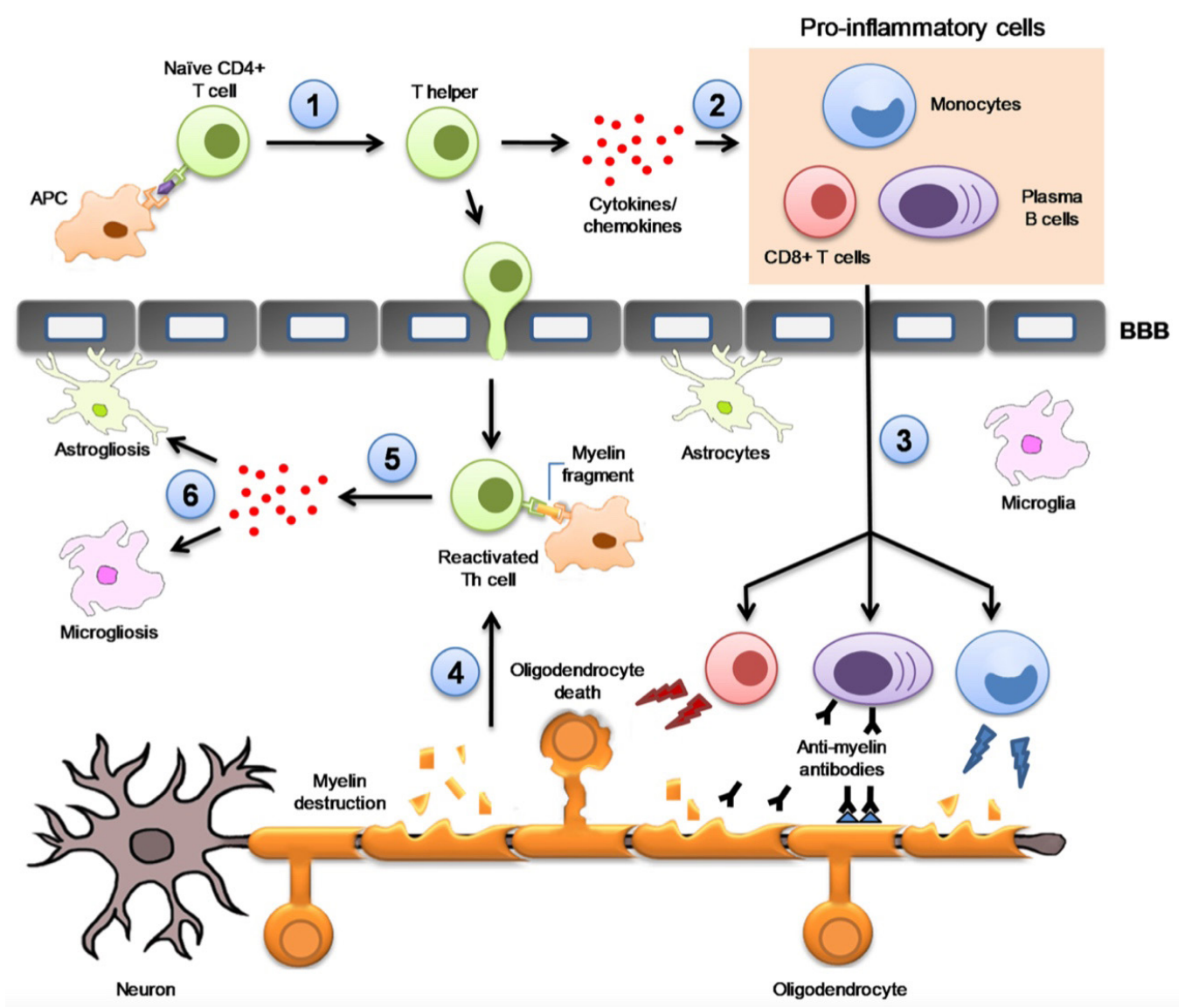

Figure 2 Key immunopathophysiological mechanisms underlying the development of MS. I) Differentiation of CD4+T naive cells into CD4+T helper cells (Th); 2) Th produces IFN- $\gamma$ to recruit CD8+T cells, B cells and monocytes in the periphery; 3) Proinflammatory cells migrate to the blood-brain barrier and pass into the CNS; 4 ) Infiltrated CD4+T cells are reactivated upon interaction with myelin fragments presented by resident APCs; 5) proinflammatory cytokines and chemokines released; 6) astrogliosis and microgliosis. Reproduced from Celarain N,Tomas-Roig J.J Neuroinflammation. 2020; 17:21. ${ }^{19}$ Open access; refer to http:// creativecommons.org/publicdomain/zero/I.0/).

APC, antigen presenting cell; BBB, blood brain barrier;Th,T helper cell. 
After interfacing with specific antigen-MHC complex and receiving costimulatory signals, activated $\mathrm{CD} 4+$ cells differentiate into proinflammatory $\mathrm{T}$ helper cells (Th1 and Th17), which release interferon gamma (INF $\gamma$ ) and tumor necrosis factor alpha (TNF $\alpha$. $\mathrm{TNF} \alpha$ causes myelin disruption and oligodendrocytes to undergo apoptosis. INF $\gamma$ is responsible for recruiting cytotoxic T lymphocytes (CD8+ cells), B lymphocytes, and monocytes, which leave the lymph nodes and migrate to the blood brain barrier (BBB) and enter the CNS. Egress of $\mathrm{T}$ cells from the lymph nodes to the blood compartment depends on the presence of sphingosine 1-phosphate receptor-1 (S1P-R1), which can be blocked by S1P-R modulators. Entry of activated $\mathrm{T}$ cells to the CNS occurs first through $\mathrm{T}$ cell crawling (margination) on the $\mathrm{BBB}$ endothelium requiring intercellular adhesion molecule-1 (ICAM-1) and then, via diapedesis, through the specialized nonfenestrated capillary endothelium. ${ }^{19}$ Diapedesis depends on specific adhesion molecules such as very late antigen-4 (VLA-4) and vascular cell adhesion molecule-1 (VCAM-1), which again represent another therapeutic target. ${ }^{23}$

Upon entry to the CNS, naïve B cells differentiate into plasma B cells and release auto-antibodies directed to CNS self-antigens and begin to attack the myelin sheath surrounding axons. However, there is increasing evidence that the role of B cells in MS is more pivotal than previously believed, an assertion bolstered by the success of B cell targeting therapies such as anti CD20. This contrasts to the longheld view that MS is primary a T cell-mediated disease. Antibodyindependent effects of B cells, via elaboration of cytokines, include antigen presentation to $\mathrm{T}$ cells, elaboration of proinflammatory cytokines (and anti-inflammatory cytokines tamping down the immune response) ${ }^{24}$

Our understanding of MS pathophysiology continues to evolve and recent evidence suggests that memory B cells, in particular, may represent a fundamental component of MS etiology, and T and B cell autoimmunity may be a secondary, rather than primary, promoter of MS. ${ }^{25,26}$ After encountering and responding to an antigen, memory B cells remain dormant until re-encounter of antigen and then rapidly react to the second challenge. Memory B cell survival can last for years independent of antigen. In MS, B cells can be found in the CNS, including the CSF, parenchyma, and meninges. ${ }^{26}$ These cells have a variety of effector functions including cytokine production and antigen presentation, and operate as antigen-experienced precursors to cells secreting antibody. ${ }^{26} \mathrm{~A}$ number of MS immunomodulatory therapies including anti-CD20 therapies (e.g., ocrelizumab), CD52 (e.g., alemtuzumab), and cladribine deplete B cells including memory cells..$^{25,27}$

Cytotoxic $\mathrm{T}$ CD8+ cells that migrate into the CNS attack oligodendrocytes, which synthesize the myelin sheath, and where they exacerbate neuronal damage. Monocytes (macrophages) entering the CNS also degrade the myelin sheath by phagocytosis and release proinflammatory cytokines that enhance the local inflammatory response. In the early stages of MS, both T CD4+ and T CD8+ cells are present in MS lesions; CD4+ T cells occur predominately in acute lesions and CD8+ T cells primarily in chronic lesions. ${ }^{28}$

The neuronal damage occurring in early lesions does not spread to surrounding brain tissue, resulting in normal-appearing white matter. In the initial stage of MS, demyelination is not necessarily permanent, and the brain has some capacity to recover through recruitment of oligodendrocyte progenitor cells to remyelinate damaged axons. ${ }^{19}$ This process of remyelination, whereby new myelin sheaths are generated around axons, is driven by several growth factors, including plateletderived growth factor (PDGF) and fibroblast growth factor (FGF). ${ }^{19}$
Remyelination becomes progressively inadequate and ultimately fails in most lesions and patients. ${ }^{29}$

The degradation of myelin sheath releases myelin fragments which, in turn, are presented by CNS resident APCs and reactivate $\mathrm{T}$ CD4+ cells resulting in release of proinflammatory cytokines and chemokines. Progression of MS is characterized by diffuse T and B cell infiltrates, astrogliosis and microgliosis, and progressive axonal loss contributing to brain atrophy and neurological disabilty. ${ }^{30}$

Neurodegeneration may also involve non-immune mechanisms such as production of oxygen and nitrogen reactive species, mitochondrial damage, impaired energy production, and intra-axonal $\mathrm{Ca}^{2+}$ accumulation that leads to catabolic enzymes activation and proteolytic degradation of cytoskeleton proteins. ${ }^{30} \mathrm{CNS}$ inflammation also releases glutamate from activated leukocytes and microglia and glutamate excitotoxicity via AMPA/kinate receptors and leads to damage to neurons and oligodendrocytes. ${ }^{23}$ Nuclear factor erythroid2-related factor 2 (Nrf2) is a central modulator of redox, metabolic, and protein homeostasis and may play a role in MS. ${ }^{31}$ Indeed, Nrf2 activation (e.g., using dimethyl fumarate or monomethyl fumarate) induces upregulation of antioxidant gene expression and is an approved therapeutic approach in relapsing types of MS. ${ }^{32}$

\section{Role of the innate immune system in MS}

The innate immune system comprises monocytes (macrophages and dendritic cells), as well as neutrophils and natural killer (NK) cells. As described above, peripheral monocytes are mobilized by cytokine signaling and cross the BBB where they are activated and differentiate into dendritic cells or macrophages (professional phagocytes). ${ }^{33}$ Macrophages not only serve as antigen-presenting cells but also play a role in both tissue destruction (M1 proinflammatory phenotype) in early MS and tissue repair (M2 phenotype) in later remission, depending on which cytokines are present. ${ }^{34}$

Although their role is not fully elucidated, NK cells may also play a role in controlling T cell activation in CNS autoimmunity, and dysfunction of NK cells may represent a contributing factor in the pathogenesis of MS. ${ }^{35}$ Neutrophils, the most abundant circulating leukocyte and the primary innate defense cells against bacteria and other micro-organisms, migrate along chemical gradients (chemotaxis) to the site of infection/injury/inflammation. Several types of chemotactic signals are responsible for neutrophil migration: bacterial formyl peptides, complement molecules C3a and C5a, and chemokines (leukotrienes and chemotactic cytokines). Evidence is accumulating that neutrophils may also play a role in MS pathogenesis by releasing inflammatory mediators and enzymes, by destroying and phagocytosing myelin debris, by releasing neutrophil extracellular traps, by producing reactive oxygen species, breaking down the BBB, and finally by generating and presenting autoantigens. ${ }^{36}$

\section{Pharmacotherapy of MS}

The past few decades have led to the development of a plethora of different types of DMDs with mechanisms of action directed at various points along MS immunopathophysiologic disease continuum. These agents, administered by self-injection, infusion or orally, are approved in the US for various types of MS, including CIS, RRMS, SPMS. Only one agent (ocrelizumab) is also indicated for PPMS (Table 2). ${ }^{5,37,38}$ In 1993, interferon (INF) $\beta$ - $1 \mathrm{~b}$ subcutaneous (SC) was the first DMD approved in the US for relapsing types of MS; the most recent addition, ponesimod, was approved for the same indications in 2021 (Figure 3). ${ }^{39}$ 
Table 2 Summary of DMDs approved in the US to treat multiple sclerosis ${ }^{5,37,38}$

\begin{tabular}{|c|c|c|}
\hline DMD & Route & Proposed Mechanism of Action \\
\hline \multicolumn{3}{|l|}{ Interferons } \\
\hline PEG-IFN $\beta$-Ia & SC, IM & $\begin{array}{l}\text { Reduces ThI and ThI7 production; promotes Th2 proliferation; regulates T-, B-, } \\
\text { NK and dendritic cells; blocks leukocyte migration to CNS }\end{array}$ \\
\hline IFN $\beta-I a$ & & “ \\
\hline IFN $\beta$-Ib & & “ \\
\hline $\begin{array}{l}\text { Glatiramer } \\
\text { acetate }\end{array}$ & SC & $\begin{array}{l}\text { Binds MHC class II; interferes with development of self-reactive } \\
\text { proinflammatory T-cells; promotes Th2 proliferation; regulates various immune } \\
\text { cells }\end{array}$ \\
\hline \multicolumn{3}{|c|}{ SIP receptor modulators } \\
\hline Siponimod & $\mathrm{PO}$ & $\begin{array}{l}\text { Blocks egress of lymphocytes from lymph node and depletes circulating } \\
\text { lymphocytes; promotes CNS repair by modulating SIPI on astrocytes and SIP5 } \\
\text { on oligodendrocytes }\end{array}$ \\
\hline Fingolimod & $\mathrm{PO}$ & $\begin{array}{l}\text { Blocks egress of lymphocytes from lymph node and depletes circulating } \\
\text { lymphocytes; reduction of lymphocyte migration into the CNS }\end{array}$ \\
\hline Ozanimod & PO & “ \\
\hline Ponesimod & $\mathrm{PO}$ & “ \\
\hline
\end{tabular}

\section{US indication}

CIS, RRMS, and active SPMS

CIS, RRMS, and active SPMS

CIS, RRMS, and active SPMS

DMF and its metabolite, monomethyl fumarate (MMF) activates Nrf2 pathway involved in cellular response to oxidative stress; Th I-Th2 shift, lymphocyte apoptosis; downregulation of $\mathrm{NF \kappa B}$

CIS, RRMS, and active SPMS

Diroximel fumarate

Activates Nrf2 pathway involved in cellular response to oxidative stress

Monomethyl

fumarate

Monoclonal antibodies

$\begin{array}{ll}\text { Ofatumumab } & \text { SC } \\ \text { Ocrelizumab } & \text { IV } \\ & \text { infusion }\end{array}$

Binds to CD20, a cell surface antigen on pre-B and mature $B$ lymphocytes; results in antibody-dependent cellular cytolysis and complement-mediated lysis

CIS, RRMS, and active SPMS

Binds to CD20, a cell surface antigen on pre-B and mature $B$ lymphocytes; results in antibody-dependent cellular cytolysis and complement-mediated lysis

CIS, RRMS, active SPMS, and PPMS

Binds to $\alpha 4$-subunit of $\alpha 4 \beta$ I and $\alpha 4 \beta 7$ integrins expressed on leukocytes (except neutrophils) and inhibits the $\alpha 4$-mediated adhesion of leukocytes to Natalizumab IV VCAM-I on vascular endothelial cells, and with CS-I and/or osteopontin expressed by parenchymal cells in the brain; prevents transmigration of leukocytes across the endothelium into inflamed parenchymal tissue

IV Binds to CD52, a cell-surface antigen present on T and B lymphocytes, and on infusion NK cells, monocytes, and macrophages; results in antibody-dependent cellular cytolysis and complement-mediated lysis

\section{Other therapies}

Mitoxantrone

Cladribine

Teriflunomide
PO

IV infusion

$\mathrm{PO}$
Inhibits type-II topoisomerase; disrupts DNA synthesis; inhibits B cell, T cell, and macrophage proliferation, impairs antigen presentation and secretion of interferon gamma, TNF $\alpha$, and IL-2

Involves cytotoxic effects on B and T lymphocytes through impairment of DNA synthesis, resulting in depletion of lymphocytes

Immunomodulatory agent with anti-inflammatory properties; pyrimidine synthesis inhibitor; reduces the number of activated lymphocytes in the CNS
RRMS, SPMS

RRMS, and active SPMS

CIS, RRMS, and active SPMS

MHC, major histocompatibility complex; CD, cluster of differentiation; CNS, central nervous system; CIS, clinically isolated syndrome; DMF, dimethyl fumarate; DNA, deoxyribonucleic acid; IL-2, interleukin-2; MMF, monomethyl fumarate; NK, natural killer; NFאB, nuclear factor kappa-light-chain-enhancer of activated B cells; Nrf2, Nuclear factor-erythroid factor 2-related factor 2; RRMS, relapsing-remitting multiple sclerosis; SPMS, secondary progressive multiple sclerosis; PPMS, primary progressive MS; PO, per os (by mouth); SC, subcutaneous; SIPI, sphingosine-I-phosphate receptor I; SIP5, sphingosine-I-phosphate receptor 5; ThI,T helper cell, type I;ThI7,T helper cell type I7;Th2,T helper cell type 2;TNF $\alpha$, tumour Necrosis Factor alpha; VCAM-I, vascular cell adhesion molecule I 


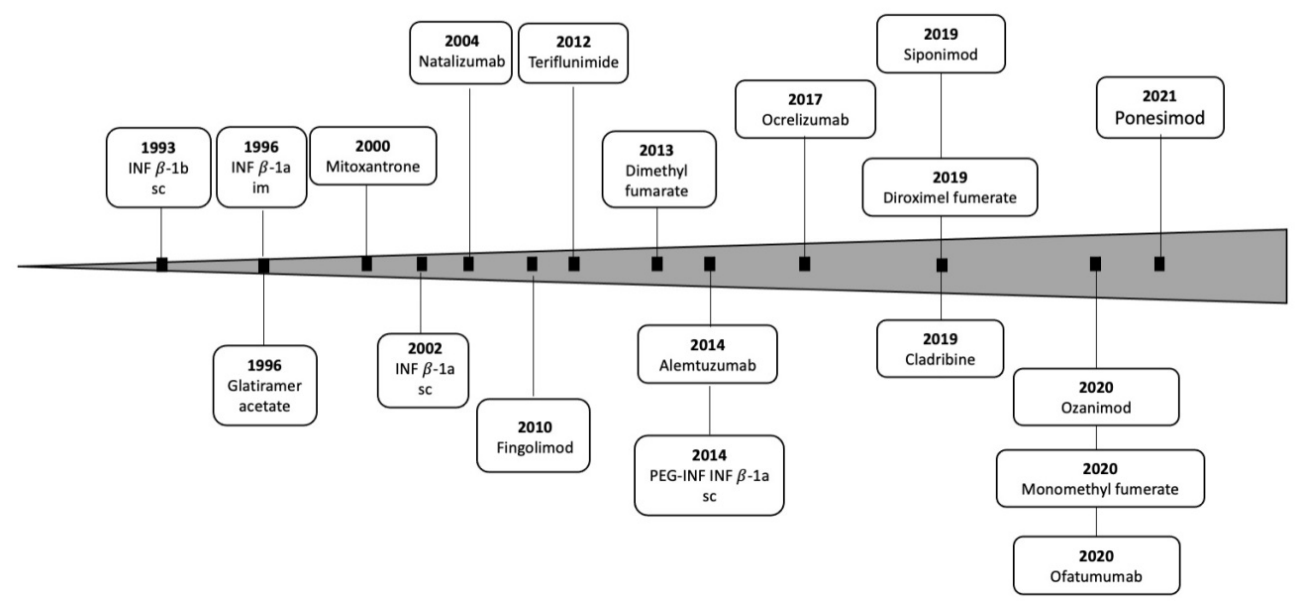

Figure 3 Timeline for approval of therapies for MS in the US. Medications commonly prescribed off label for the treatment of MS were omitted. Based in part on Melamed E and Lee MW. Front Immunol. 2020;10:2954. ${ }^{39}$

While a wide range of therapies proffers a more individualized treatment approach, the question becomes which treatment best suits a specific patient, and whether or not a therapy should be switched due to lack of efficacy or difficulty tolerating. This decision should take into account a number of factors such as patient characteristics (e.g., comorbidities), disease activity/severity, drug safety profile, and drug efficacy, benefit: risk profile, route of administration, and monitoring requirements. ${ }^{40}$ It is important to note that all agents may have adverse effects, ranging from bothersome to life-threatening, and the risks must be balanced with efficacy in individual patients.

Ideally, DMD treatment should be initiated early in the disease course with the goal to reduce disease activity, preserve brain tissue, and limit deterioration in cognitive and physical function. ${ }^{3}$ The efficacy of current DMDs for MS offer clinicians the possibility to halt disease progression..$^{41}$ At least in the clinical trial setting, no evidence of disease activity (NEDA) represents a valuable tool to assess DMD efficacy. NEDA is a combined assessment involving three individual domains (no relapses, no 3-month sustained change in expanded disability status scale (EDSS) score, and no new MRI lesions (no T1 Gd-enhancing or active T2 lesions). ${ }^{41,42}$

In addition, there are a plethora of supportive care agents to treat symptoms associated with MS. These include, for example, therapies to treat bladder problems, bowel dysfunction, depression, vertigo/ dizziness, emotional changes, fatigue, itching, pain, sexual problems, spasticity, tremors, and gait difficulties. However, these symptomdirected therapies are beyond the scope of this article.

DMDs can also be differentiated according to overall treatment concept: that is, chronic maintenance/escalation therapy and immune reconstitution therapy or IRT (Figure 4). ${ }^{37,43}$ IRT was originally used to describe immunological outcomes of allogenic and autologous hematopoietic stem cell transplantations (HSCT) whereby the repertoire of adaptive immune cells was reestablished. The overarching goal being the elimination of pathogenic immune cells via immune cell depletion and repopulate with new cells offering immune tolerance. ${ }^{44}$ IRT, also referred to immune depletion and repopulation therapy, is characterized by a short course of therapy either as a single therapy (e.g., autologous HSCT or intermittent/short course, e.g., cladribine and alemtuzumab) followed by treatment-free periods. ${ }^{44}$ IRT has the potential to induce longer-term remission that goes beyond the short course dosing period. ${ }^{37}$ It remains unclear, however, whether IRT agents such as alemtuzumab and cladribine achieve a reset of the adaptive immune system or simply a consequence of different dynamics of repopulation for different cell types. ${ }^{44}$

For example, alemtuzumab is effective in achieving NEDA after a short course an immune depleting therapy with three mechanisms of action: 1) targets CD52+ on $\mathrm{T}$ and $\mathrm{B}$ cells (and innate immune cells, albeit to a minor extent) 2) rapid depletion of immune cells, and 3) a phase of repopulation. ${ }^{45}$ The repopulation phase is characterized by a rapid hyper-repopulation of immature $\mathrm{B}$ cells but sustained depletion of memory B cells, which are postulated to be key drivers of MS disease progression. With respect to cladribine, a sub-study of MAGNIFY-MS MRI, an ongoing Phase IV open-label 2-year study, longitudinally evaluated changes in peripheral adaptive immune cell subtypes after receiving cladribine. The results suggested that the effects of cladribine action may, in part, result from a depletion of memory B cells for at least 12 months, a moderate decrease across $\mathrm{T}$ cell subtypes, and a recovery over baseline for regulatory B cells, effects that occur without persistent immunosuppression. ${ }^{46}$

In comparison, maintenance therapy is administered continuously and/or escalated over time with changes in immune function occurring only during active dosing. The latter therapy can also be further subdivided into immunomodulatory or immunosuppressive. An immunosuppressive DMD (either continuous or intermittent) reduces the activation of the immune system and causes notable lymphopenia and, depending on the duration of therapy, may also be associated with opportunistic infections, reduced vaccine responsiveness, and risk of secondary malignancies. ${ }^{37,47}$ Examples of continuous immunosuppressive agents include: dimethyl fumarate, fingolimod, ocrelizumab, natalizumab, and teriflunomide. With respect to immunomodulators, the overall activity of the immune system is not suppressed per se but is modulated at different points, including reduction of inflammatory mediators and activation and proliferation of lymphocytes and entry into the CNS. Examples of immunomodulators of relapsing MS include interferon and glatiramer acetate. ${ }^{47}$

A number of clinical practice guidelines are available, including those from European Committee for Treatment and Research in Multiple Sclerosis (ECTRIMS)/European Academy of Neurology (EAN), ${ }^{48}$ the American Academy of Neurology (AAN),${ }^{40} \mathrm{UK}$ National Institute for Health and Care Excellence (NICE), ${ }^{49}$ and the German Neurological Society Guidelines on Diagnosis and Treatment of MS..$^{50}$ The AAN guidelines are based on a comprehensive systematic review 
of over 50 clinical trials in MS that have occurred primarily over the past two decades. The guidelines address 17 statements relating to initiating treatment (10 statements regarding treatment switches, and 3 statements on stopping DMTs) and are a valuable resource in navigating the use of the multiple treatments available for MS.

Treatment concept

\begin{tabular}{|c|c|c|c|}
\hline MS severity & $\begin{array}{c}\text { Chronic/maintenance } \\
\text { therapies }\end{array}$ & $\longleftrightarrow \begin{array}{c}\text { Pulsed immune } \\
\text { reconstitution therapies }\end{array}$ & MS prognosis \\
\hline $\begin{array}{l}\text { Malignant } \\
\text { Refractory } \\
\text { Aggressive }\end{array}$ & Natalizumab & $\begin{array}{c}\text { HSCT } \\
\text { Alemtuzumab } \\
\text { (anti-CD52) }\end{array}$ & Poor \\
\hline Higniy active & Fingolimod & Cladribine & \multirow{3}{*}{ Indeterminate } \\
\hline Active & 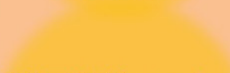 & $\begin{array}{l}\text { Ocrelizumab } \\
\text { (anti-CD20) }\end{array}$ & \\
\hline Moderate & $\begin{array}{l}\text { Dimethyl fumarate } \\
\text { Glatiramer acetate }\end{array}$ & & \\
\hline Mild & $\begin{array}{l}\text { Interferons } \\
\text { Teriflunomide }\end{array}$ & & \multirow[t]{3}{*}{ Favorable } \\
\hline Inactive & & & \\
\hline & No & Yes & \\
\hline
\end{tabular}

Figure 4 Overview of different MS measures in trajectory to treatment concepts. Reproduced with permission from Wiendl, H. Nat Rev Neurol. 20I7;13:573$574 .{ }^{43}$

HSCT, hematopoietic stem cell transplantation.

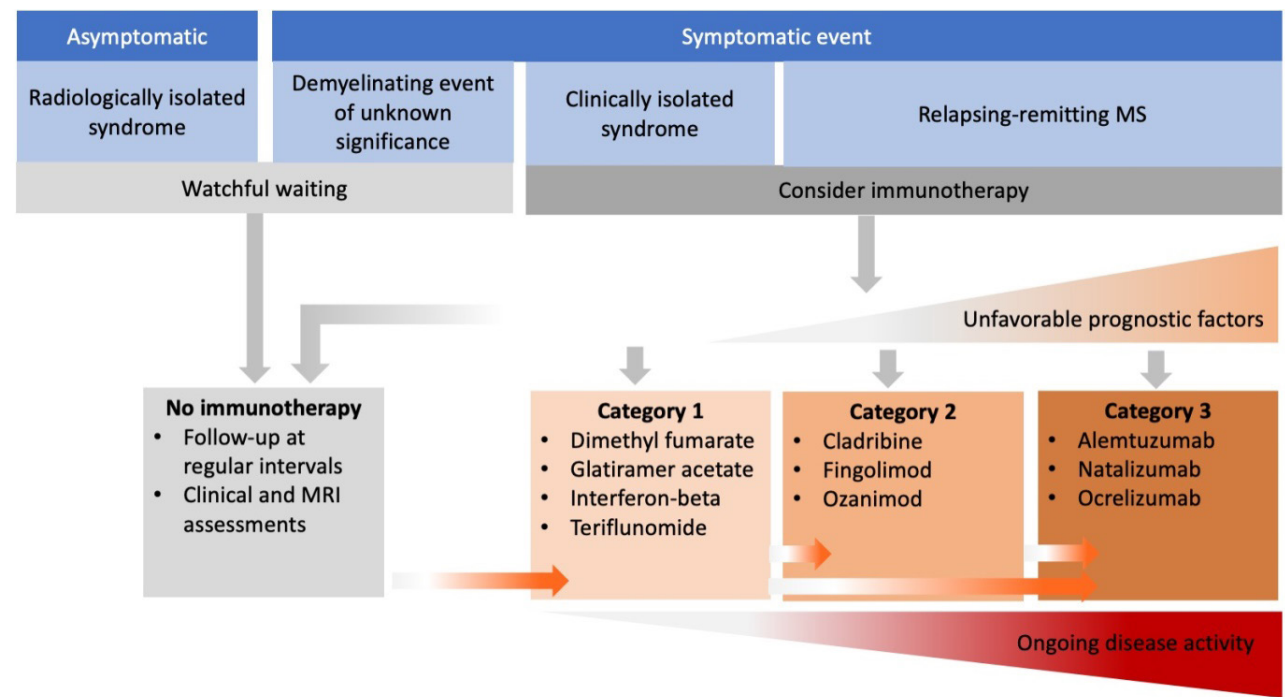

Figure 5 Summary of the selection and timing of DMTs to treat relapsing MS. Reproduced from Bayas A et al. Neurological Research and Practice. 202I;6;3(I):45. ${ }^{50}$ Open access; refer to https://creativecommons.org/licenses/by/4.0/).

A simplified overview of the selection and timing of DMDs to treat relapsing MS is portrayed in Figure $5 .{ }^{50}$ Three categories of efficacy drugs are presented: Efficacy category 1 (INF-beta, dimethyl fumerate, glatiramer acetate, and teriflunomide), category 2 (cladribine, fingolimod, and ozanimod) and category 3 (alumtuzumab, anti-CD20 antibodies including ocrelizumab, rituximab, and natalizumab). The selection of DMDs should be individualized for each patient based on disease activity, relapse frequency and severity, response to relapse therapy, disease progression, and MRI findings. This treatment approach proposes that category 1 drugs be used as first line treatment in DMD-naïve patients unless the patient is experiencing highly active disease. Categories 2 and 3 should be considered if category 1 drugs have failed to control the disease or if DMD-naïve patients are considered to have highly active disease from the onset.

Guidelines are constantly changing in the light of new clinical and scientific findings. Therefore, the most current, countryspecific manufacturer's product information should be consulted for indications, recommended dosing, duration of therapy, and contraindications. When prescribing any product mentioned in this 
article, the individual practitioner should integrate their personal clinical experience and patient values with the best clinical evidence in making decisions about the care of individual patients.

\section{Conclusions}

Immunopathophysiological mechanisms underlying the development of MS are complex and are only partially delineated. Currently, the underlying key pathways can be summarized as follows: 1) Autoreactive $\mathrm{T}$ cells that have eluded central tolerance in the thymus enter the lymph nodes, 2) APCs, including B cells, expressing an antigen closely resembling part of a self-protein or putative autoantigens, activate CD4+ cells, 3) Differentiation of CD4+ naïve cells into T helper cells, 4) T helper cells produce IFN- $\gamma$ to recruit T CD8+ cells, B cells and monocytes in the periphery, 5) Proinflammatory cells migrate into the CNS, 6) Infiltrated CD4+ cells are resurrected upon interaction with myelin fragments presented by resident APCs, 7) proinflammatory cytokines and chemokines are released; 8) astrogliosis and microgliosis ensue. Ongoing research continues to reveal new aspects of this disease and it is becoming clear that B cells, particularly memory B cells, play a more central role than previously believed. Understanding the immunopathological basis for MS has revolutionized the treatment of this neurological autoimmune disease with a plethora of DMDs with mechanisms of action directed at various points along MS immunopathophysiologic disease continuum. Many more potential therapeutic approaches are in clinical development, including Bruton's tyrosine kinase inhibitors, cell-based therapies, remyelination, immune tolerance, neuroprotection (e.g., targeting mitochondria), and modulation microbiome triggers..$^{14,51-54}$

\section{Funding}

The author received no funding to develop this article.

\section{Acknowledgments}

None.

\section{Conflicts of interest}

The author previously served as an external reviewer of medical and scientific materials for immunology and neurology at EMD Serono.

\section{References}

1. Zalc B. One hundred and fifty years ago Charcot reported multiple sclerosis as a new neurological disease. Brain. 2018;141(12):3482-3488.

2. Who gets MS? Epidemiology. National Multiple Sclerosis Society.

3. Giovannoni G, Butzkueven H, Dhib-Jalbut $\mathrm{S}$, et al. Brain health: time matters in multiple sclerosis. Mult Scler Relat Disord. 2016;9 Suppl 1:S5S48.

4. National Multiple Sclerosis Society. Updated atlas of MS shows over 2.8 million people worldwide have multiple sclerosis-with nearly 1 million in the US; 2020.

5. Baecher-Allan C, Kaskow BJ, Weiner HL. Multiple sclerosis: Mechanisms and immunotherapy. Neuron. 2018;97(4):742-768.

6. Noseworthy JH, Lucchinetti C, Rodriguez M, et al. Multiple sclerosis. $N$ Engl J Med. 2000;343(13):938-952.

7. Dendrou CA, Fugger L, Friese MA. Immunopathology of multiple sclerosis. Nat Rev Immunol. 2015;15(9):545-558.

8. Ghasemi N, Razavi S, Nikzad E. Multiple sclerosis: Pathogenesis, symptoms, diagnoses and cell-based therapy. Cell J. 2017;19(1):1-10.
9. Doshi A, Chataway J. Multiple sclerosis, a treatable disease. Clin Med (Lond). 2016;16(Suppl 6):s53-s59.

10. Hauser SL, Oksenberg JR. The neurobiology of multiple sclerosis: genes, inflammation, and neurodegeneration. Neuron 2006;52(1):61-76.

11. Weiner HL. The challenge of multiple sclerosis: how do we cure a chronic heterogeneous disease? Ann Neurol. 2009;65(3):239-248.

12. Ziemssen T, Derfuss T, de Stefano N, et al. Optimizing treatment success in multiple sclerosis. J Neurol. 2016;263(6):1053-1065.

13. Jack B. MS: The treatment paradigm, A pathway to success for improved patient outcomes. J Managed Care Med. 2009;12(1):29-31.

14. Ransohoff RM, Hafler DA, Lucchinetti CF. Multiple sclerosis-a quiet revolution. Nat Rev Neurol. 2015;11(3):134-142.

15. Hemmer B, Kerschensteiner M, Korn T. Role of the innate and adaptive immune responses in the course of multiple sclerosis. Lancet Neurol. 2015;14(4):406-419.

16. Burks J. MS: The treatment paradigm, a pathway to success for improved patient outcomes. J Managed Care Med. 2009;12(1):26-31.

17. Strasser A, Puthalakath H, O'Reilly LA, et al. What do we know about the mechanisms of elimination of autoreactive $\mathrm{T}$ and $\mathrm{B}$ cells and what challenges remain. Immunol Cell Biol. 2008;86(1):57-66.

18. Cvetanovich GL, Hafler DA. Human regulatory T cells in autoimmune diseases. Curr Opin Immunol. 2010;22(6):753-760.

19. Celarain N, Tomas-Roig J. Aberrant DNA methylation profile exacerbates inflammation and neurodegeneration in multiple sclerosis patients. $J$ Neuroinflammation. 2020;17(1):21.

20. Waubant E, Lucas R, Mowry E, et al. Environmental and genetic risk factors for MS: an integrated review. Ann Clin Transl Neurol. 2019;6(9):1905-1922.

21. Jakimovski D, Guan Y, Ramanathan M, et al. Lifestyle-based modifiable risk factors in multiple sclerosis: review of experimental and clinical findings. Neurodegenerative Disease Management. 2019;9(3):149-172.

22. Ireland S, Monson N. Potential impact of B cells on T cell function in multiple sclerosis. Mult Scler Int. 2011;2011:423971.

23. Zamvil SS, Steinman L. Diverse targets for intervention during inflammatory and neurodegenerative phases of multiple sclerosis. Neuron. 2003;38(5):685-688.

24. Sabatino JJ, Probstel AK, Zamvil SS. B cells in autoimmune and neurodegenerative central nervous system diseases. Nat Rev Neurosci. 2019;20:728-745

25. Baker D, Marta M, Pryce G, et al. Memory B cells are major targets for effective immunotherapy in relapsing multiple sclerosis. EBioMedicine. 2017;16:41-50

26. DiSano KD, Gilli F, Pachner AR. Memory B cells in multiple sclerosis: Emerging players in disease pathogenesis. Front Immunol. 2021;12:676686.

27. Ceronie B, Jacobs BM, Baker D, et al. Cladribine treatment of multiple sclerosis is associated with depletion of memory B cells. J Neurol. 2018;265(5):1199-1209.

28. Chitnis T. The role of CD4 T cells in the pathogenesis of multiple sclerosis. Int Rev Neurobiol. 2007;79:43-72.

29. Chari DM. Remyelination in multiple sclerosis. Int Rev Neurobiol. 2007;79:589-620.

30. Correale J, Marrodan M, Ysrraelit MC. Mechanisms of neurodegeneration and axonal dysfunction in progressive multiple sclerosis. Biomedicines. 2019;7(1):14

31. Dodson M, de la Vega MR, Cholanians AB, et al. Modulating NRF2 in disease: Timing is everything. Annu Rev Pharmacol Toxicol. 2019;59:555-575. 
32. Brandes MS, Gray NE. NRF2 as a Therapeutic target in neurodegenerative diseases. ASN Neuro. 2020;12:1759091419899782.

33. Nally FK, De Santi C, McCoy CE. Nanomodulation of macrophages in multiple sclerosis. Cells. 2019;8(6):543.

34. Chu F, Shi M, Zheng C, et al. The roles of macrophages and microglia in multiple sclerosis and experimental autoimmune encephalomyelitis. $J$ Neuroimmunol. 2018;318:1-7.

35. Gross CC, Schulte-Mecklenbeck A, Wiendl H, et al. Regulatory functions of natural killer cells in multiple sclerosis. Front Immunol. 2016;7:606

36. De Bondt $\mathrm{M}$, Hellings $\mathrm{N}$, Opdenakker $\mathrm{G}$, et al. Neutrophils: Underestimated players in the pathogenesis of multiple sclerosis (MS). Int J Mol Sci. 2020;21(12):4558.

37. Giovannoni G. Disease-modifying treatments for early and advanced multiple sclerosis: a new treatment paradigm. Curr Opin Neurol. 2018;31(3):233-243.

38. Zhang, L, Tian JY, Li B. Current immunotherapies for multiple sclerosis and neuromyelitis optica spectrum disorders: the similarities and differences. Neuroimmunol Neuroinflammation. 2019;6:8.

39. Melamed E, Lee MW. Multiple sclerosis and cancer: The Ying-Yang effect of disease modifying therapies. Front Immunol. 2020;10:2954.

40. Rae-Grant A, Day GS, Marrie RA, et al. Practice guideline recommendations summary: Disease-modifying therapies for adults with multiple sclerosis: Report of the Guideline Development, Dissemination, and Implementation Subcommittee of the American Academy of Neurology. Neurology. 2018;90(17):777-788.

41. Giovannoni G, Tomic D, Bright JR, et al. "No evident disease activity": The use of combined assessments in the management of patients with multiple sclerosis. Mult Scler. 2017;23(9):1179-1187.

42. Giovannoni G, Cook S, Rammohan K, et al. Sustained disease-activityfree status in patients with relapsing-remitting multiple sclerosis treated with cladribine tablets in the CLARITY study: a post-hoc and subgroup analysis. Lancet Neurol. 2011;10(4):329-337.

43. Wiendl H. Cladribine-an old newcomer for pulsed immune reconstitution in MS. Nat Rev Neurol. 2017;13(10):573-574.
44. Lunemann JD, Ruck T, Muraro PA, et al. Immune reconstitution therapies: concepts for durable remission in multiple sclerosis. Nat Rev Neurol. 2020;16(1):56-62.

45. Wiendl H, Bourdette D, Ciccarelli O. Can immune reprogramming with alemtuzumab induce permanent remission in multiple sclerosis? Neurology. 2017;89(11):1098-1100.

46. Wiendl H, Schmierer K, Hodgkinson S, et al. Characterization of peripheral immune cell dynamics and repopulation patterns in the first 12 months of cladribine tablets treatment: MAGNIFY-MS Study. AAN Virtual Congress; 2021.

47. AlSharoqi IA, Aljumah M, Bohlega S, et al. Immune reconstitution therapy or continuous immunosuppression for the management of active relapsing-remitting multiple sclerosis patients? A Narrative Review. Neurol Ther. 2020;9(1):55-66.

48. Montalban X, Gold R, Thompson AJ, et al. ECTRIMS/EAN Guideline on the pharmacological treatment of people with multiple sclerosis. Mult Scler. 2018;24(2):96-120.

49. National Institute for Health and Care Excellence (NICE) guidance. Multiple sclerosis; 2021.

50. Bayas A, Berthele A, Hemmer B, et al. Controversy on the treatment of multiple sclerosis and related disorders: positional statement of the expert panel in charge of the 2021 DGN Guideline on diagnosis and treatment of multiple sclerosis, neuromyelitis optica spectrum diseases and MOG-IgGassociated disorders. Neurol Res Pract. 2021;3:45.

51. Esmaeil Amini M, Shomali N, Bakhshi A, et al. Gut microbiome and multiple sclerosis: New insights and perspective. Int Immunopharmacol. 2020;88:107024.

52. Piehl F. Current and emerging disease-modulatory therapies and treatment targets for multiple sclerosis. J Intern Med. 2021;289(6):771-791.

53. Kammona O, Kiparissides C. Recent advances in antigen-specific immunotherapies for the treatment of multiple sclerosis. Brain Sci. 2020;10(6):333.

54. Campbell G, Licht-Mayer S, Mahad D. Targeting mitochondria to protect axons in progressive MS. Neurosci Lett. 2019;710:134258. 